\title{
Faloplastia con colgajo anterolateral del muslo regional. Experiencia inicial de un caso
}

\author{
Carolina María Posso-Zapata', Daniel Vélez-Restrepo² ${ }^{2}$ Natali Durán-Casado ${ }^{3}$, Juan Pablo Hidalgo-Moncada4
}

\section{RESUMEN}

La faloplastia es un procedimiento quirúrgico reconstructivo que tiene dentro de sus objetivos alcanzar un buen resultado funcional y estético, mediante la transferencia de tejido que ofrezca sensibilidad táctil y erógena, la micción de pie y la penetración durante las relaciones sexuales. En este reporte se presenta la experiencia inicial en la IPS Universitaria Sede Clínica León XIII, con un caso de amputación traumática completa usando el colgajo anterolateral del muslo de forma regional. En este paciente se logró la reconstrucción completa del defecto, el colgajo sobrevivió en un 100 \% y sólo se presentó una pequeña pérdida del injerto de piel en la zona donante como complicación menor, que no requirió ningún procedimiento adicional. En el caso presentado se usó un método que permite la reconstrucción del falo en un solo tiempo quirúrgico, sin necesidad de microcirugía, con poca morbilidad en el sitio donante y altas tasas de éxito.

\section{PALABRAS CLAVE}

Amputación; Cologajos Quirúrgicos; Pene; Reconstrucción

\footnotetext{
1 Porfesora Asociada Sección de Cirugía Plástica, Facultad de Medicina, Universidad de Antiquia, Medellín, Colombia

2 Residente de Cirugía Plástica de cuarto año, Universidad de Antioquia, Medellín, Colombia.

3 Residente de Cirugía Plástica de tercer año, Universidad de Antioquia, Medellín, Colombia.

4 Estudiante de Medicina, Universidad de Antioquia, Medellín, Colombia.
}

Correspondencia: Carolina María Posso-Zapata; carolina.posso@gmail.com Recibido: julio 10 de 2018

Aceptado: septiembre 24 de 2018

Cómo citar: Posso-Zapata CM, Vélez-Restrepo D, Durán-Casado N, Hidalgo-Moncada JP. Faloplastia con colgajo anterolateral del muslo regional. Experiencia inicial de un caso. latreia. 2019 Abr-Jun; 32(2):147-152. D0I 10.17533/udea.iatreia.05. 


\section{SUMMARY}

\section{Phalloplasty using the pedicled anterolateral thigh flap: initial experience in one case}

phalloplasty is a reconstructive procedure, whose main objective is to achieve a good functional and aesthetic result, using local tissue to get tactile and erogenous sensibility, standing urination and penetration during sexual intercourse. In this report, we present the initial experience at the IPS Universitaria León XIII Clinic, with a clinical case of complete traumatic amputation, using the anterolateral thigh flap as a local flap. On this patient, we were able to reconstruct the defect completely, the flap survival was $100 \%$, and as a minor complication there was a partial skin graft loss, but no additional surgical treatment was required. The regional used of the anterolateral thigh flap is a reliable surgical method to reconstruct complete penile defects, in one surgical time, without the use or need of microsurgery, with minimal morbility and high rates of success.

\section{KEY WORDS}

Amputation; Penis; Reconstruction; Surgical Flaps

\section{INTRODUCCIÓN}

La faloplastia o reconstrucción del pene es un reto complejo en cirugía plástica. Las malformaciones congénitas, traumas, el cáncer o la ausencia, parcial o total, del pene, producen déficits funcionales y psicológicos, incluyendo la incapacidad para la micción de pie y las relaciones sexuales, por lo que es necesario su reconstrucción $(1,2)$. La faloplastia está indicada, además, de forma más reciente en el contexto de pacientes con trastorno de identidad de género $(1,2)$.

En el último siglo, los intentos reconstructivos se han visto limitados por la imposibilidad de encontrar un tejido que reemplace el tejido eréctil y uretral, por lo cual la reconstrucción de un pene totalmente funcional no ha sido exitosa a la fecha $(1,2)$.

Actualmente, el colgajo libre antebraquial radial es considerado el de elección para la reconstrucción peneana cuando se habla de un defecto completo. Sin embargo, dentro de sus desventajas se encuentran la importante morbilidad en la zona donante y los estigmas sociales derivados de la misma, el sacrificio de un eje vascular mayor en una extremidad, la atrofia del colgajo con el tiempo, la presencia de unidades pilosas, la posibilidad de cálculos uretrales (3), las diferencias en el color de la zona donante y receptora y la necesidad del recurso microquirúrgico (2). Por las razones previamente expuestas, algunos pacientes rechazan este procedimiento y hacen necesario considerar opciones regionales dentro de las posibilidades reconstructivas. Con el desarrollo de los colgajos perforantes, el colgajo anterolateral del muslo (ALM) ha surgido como una opción válida y ha ganado aceptación con resultados favorables a larogo plazo, mostrando menor morbilidad en el área donante, con una cicatriz fácil de ocultar y pudiendo ser realizada por cualquier cirujano plástico, aun en instituciones donde no se disponga del recurso de la microcirugía, situación que en el contexto de nuestro medio es bastante relevante $(4,5)$.

El objetivo de esta publicación es presentar la experiencia inicial con un caso de faloplastia en un paciente con amputación traumática completa del pene y ambos testículos, en el que se logró la reconstrucción exitosa con un colgajo ALM perforante en un solo tiempo quirúrogico.

\section{CASO CLÍNICO}

Paciente masculino de 37 años de edad, con antecedente de esquizofrenia paranoide sin manejo farmacológico regular, ya que habita en una región rural del departamento de Antioquia. Se dio un tratamiento inicial del trauma por el servicio de Urología, se evidenció una amputación del tercio proximal con exposición de cuerpos cavernosos y de la uretra, además de la ausencia completa del escroto y ambos testículos. Se realiza de forma inicial por Urología, desbridamiento, cierre de cuerpos cavernosos, espatulación de la uretra, vasectomía bilateral y eversión de la mucosa uretral. Posteriormente, se hizo el manejo inicial de la herida con curaciones y tratamiento antibiótico. De igual forma, desde el ingreso se contó con el apoyo del servicio de siquiatría, quien ajusta la medicación para el control del episodio sicótico, y se apoyó en sicología para el acompañamiento del paciente $y$ el control de ideas de autoagresión y alucinaciones. 
Después de 16 días, se solicita la valoración por el grupo de cirugía plástica por presentarse un defecto de cobertura de la zona y con el objetivo de restaurar la micción de pie que evite el uso permanente de sonda vesical al paciente.

Luego del manejo inicial, se evidenció un defecto de cobertura circunferencial sobre el muñón residual del pene de $5 \mathrm{~cm}$ de diámetro y la ausencia del falo, que obligaba al paciente el uso definitivo de sonda vesical, por esta razón se consideró necesario realizar la reconstrucción peneana (Figura 1, A). De las opciones disponibles, se decidió realizar la reconstrucción con colgajo anterolateral del muslo, ya que implicaba la menor morbilidad, evitaba el uso de la microcirugía, un menor tiempo quirúrgico y un uso más racional de los recursos de la institución, en especial para el caso del paciente en donde los objetivos son estrictamente funcionales por su comorbilidad siquiátrica de base.
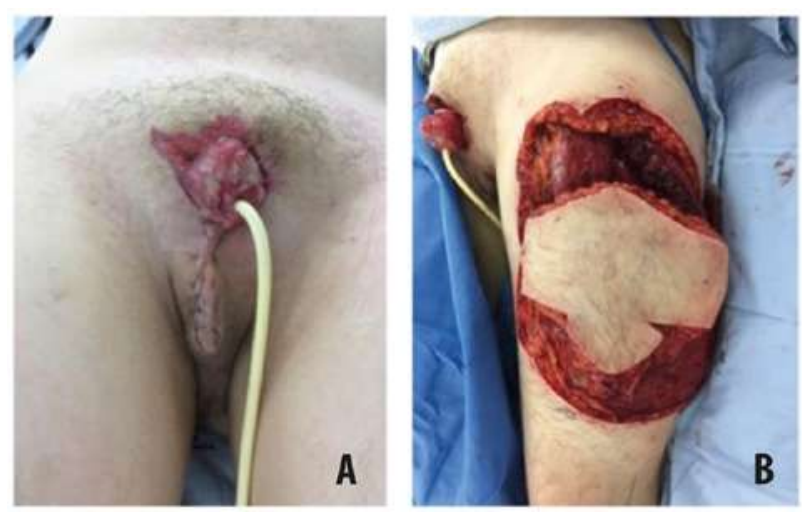

Figura 1. A: defecto preoperatorio. B: diseño intraoperatorio del colgajo regional anterolateral del muslo, se muestra diseño de la isla

En un primer momento quirúrogico, se realizó de parte de Urología meatoplastia y cambio de sonda vesical. Continúa cirugía plástica continua con el proceso de reconstrucción así: marcación de perforantes en el muslo izquierdo y diseño de colgajo anterolateral de muslo (ALM) según diseño de Morrison et al. (Figura 1, B), previo a la asepsia general. Es de anotar que la longitud y el diseño del glande fueron simplificados, teniendo presente los objetivos de la reconstrucción del caso en particular. Durante la disección del colgajo con el equipo de cirugía vascular y magnificación con lupas 2,5x, se logró identificar 2 perforantes musculocutáneas con trayecto sobre el músculo vasto lateral, por lo cual fue necesario disecarlas hasta su emergencia como ramas de la arteria circunfleja femoral lateral y seguidamente, esta última arteria se disecó hasta su emergencia en la arteria femoral profunda, para lograr así la mayor longitud total del pedículo. A continuación, se hace la transposición del colgajo fasciocutáneo (isla cutánea de $15 \times 18 \mathrm{~cm}$ ) por debajo del músculo recto femoral, adicionando miotomía del sartorio para evitar la compresión del pedículo.

Se incluyó además en el diseño del colgajo el nervio cutáneo femoral anterior y se realizó anastomosis primaria con nervio pudendo dorsal superficial, con el fin de lograr una recuperación sensitiva de protección. El cierre del sitio donante se realizó por avance de colgajos locales e injertos de piel de espesor parcial en el defecto residual central. El postoperatorio transcurrió sin complicaciones vasculares del colgajo, se presentó una pérdida parcial del injerto de piel del muslo donante, el cual luego de 30 días presentó cicatrización completa con curaciones (Figura 2, A). Se logró el control de la enfermedad de base con antipsicóticos y se dio de alta sin complicaciones.

En el seguimiento final, se evidenció que el colgajo perdió al menos un $30 \%$ de su volumen y longitud, pero cumplió con los objetivos funcionales propuestos inicialmente (Figura 2, B). EI paciente realiza micción de pie y se encuentra satisfecho con el aspecto del neofalo, no ha presentado recaídas o nuevas alucinaciones con la medicación iniciada desde la hospitalización.

\section{DISCUSIÓN}

La faloplastia como procedimiento reconstructivo ha incrementado su frecuencia en los últimos años, principalmente por el aumento de los casos de reasiognación de sexo (femenino, masculino), que pueden ascender hasta los 25 millones por año en el mundo $(1,2)$. Aunque también contribuyen las resecciones oncológicas, la gangrena de Fournier y las amputaciones traumáticas, todas estas entidades implican la ausencia del pene, una condición con gran impacto físico y psicológico que demanda un plan reconstructivo exigente (1). 

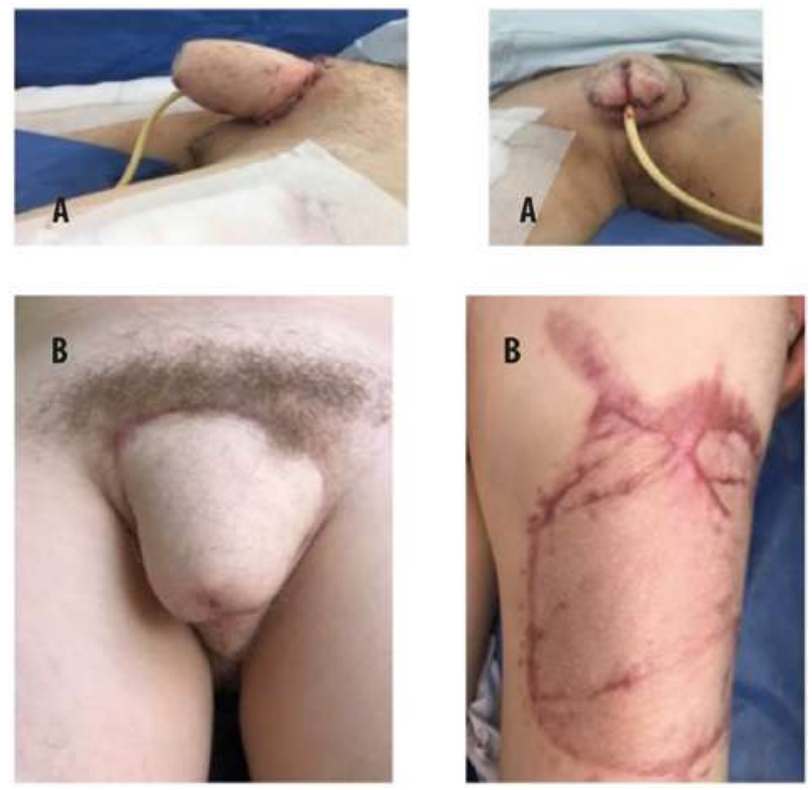

Figura 2. A: resultado postoperatorio inicial vista basal y lateral: se muestra la longitud y el volumen restaurado, la reconstrucción de la uretra y la apariencia del neoglande. B: Resultado al final del seguimiento

El espectro de posibilidades reconstructivas varía según la necesidad del tejido a reparar (pene, escroto, uretra), la anatomía vascular local disponible y la habilidad del cirujano. Borgoras en 1936, fue el primero en realizar una faloplastia utilizando un colgajo abdominal tubulizado (2). Posteriormente, Gillies y Millard en 1957, publicaron su modificación con el concepto de "tubo dentro de tubo" $(2,3)$, el cual se utiliza hasta la fecha.

A partir de estos primeros esfuerzos reconstructivos, se han desarrollado múltiples colgajos tanto regionales como libres, siendo a la fecha el colgajo antebraquial radial libre el más utilizado (4-7). Este colgajo permite la transferencia de tejido poco bultoso, logrando longitudes del neofalo entre 7,5 y $14 \mathrm{~cm}$ (1). Además, permite la recuperación táctil y erógena al preservar el nervio cutáneo antebraquial medial y/o lateral, para la posterior anastomosis con el nervio ilioinguinal o nervio dorsal del pene (6).

EI colgajo ALM fue descrito por primera vez por Song en 1984 (4). Fue utilizado como colgajo libre en 2006 para la reconstrucción peneana en 6 pacientes transgénero (1). Sus ventajas incluyen el hecho de ser un colgajo sensible, permite alcanzar longitudes de hasta $10 \mathrm{~cm}$ con un diámetro de $3,5 \mathrm{~cm}$, ofrece una forma y consistencia adecuadas, facilita la reconstrucción uretral en el mismo tiempo quirúrgico, cuenta con un pedículo vascular largo, es un tejido de volumen adecuado, con color similar al sitio receptor, deja una cicatriz fácil de ocultar en el sitio donante y presenta estabilidad del volumen en el seguimiento a largo plazo. Como principales desventajas destacan la presencia de tejido graso abundante en personas con IMC mayores de $30 \mathrm{~kg} / \mathrm{m}^{2}$, lo cual se traduce en dificultades técnicas durante su realización y la necesidad de hacer uso de la microcirugía tanto para la anastomosis vascular como para la anastomosis del nervio cutáneo femoral al nervio dorsal del pene, cuando se realiza como colgajo libre (8).

Aunque al final del seguimiento del caso presentado no se evidenció la presencia de fístulas, se especula que la incidencia de esta complicación y de dehiscencias con el colgajo ALT es mayor con respecto al colgajo antebraquial radial, lo cual posiblemente está en relación con el volumen del colgajo (9). En 2017 (7), se compararon los desenlaces del colgajo antebraquial radial con el del colgajo ALM regional, encontrando para este último mayores tasas de pérdidas parciales o totales, mayor incidencia de fístulas uretrales (OR 2,5), complicaciones no uretrales (OR 2,38) y dehiscencia (OR 5,03).

En la búsqueda de menores tasas de complicaciones y en la ausencia del recurso microquirúrogico, se ha utilizado el colgajo ALM regional, manteniendo las ventajas descritas previamente del colgajo libre $(1,6-8)$ y, adicionando la disminución del tiempo quirúrgico (3:9). En la Tabla 1, presentamos las principales series de caso descritas en la literatura con los resultados obtenidos y el tamaño de la isla cutánea del colgajo, en donde es evidente que la mayoría son reportes de casos, excepto por la serie de Rashid et al. (2010), en donde se reporta una tasa de falla o necrosis parcial de los colgajos del $0 \%(9,10)$. Otra de las consideraciones en este tipo de reconstrucción tiene que ver con la transposición del mismo, ya que existen varias técnicas cuando se usa como colgajo regional, entre las cuales está la forma submuscular al recto femoral o los aductores del muslo (5). En nuestra experiencia consideramos que es suficiente la transposición por debajo del sartorio y el recto femoral para no alterar 
la anatomía de la zona, evitando además la compresión del pedículo vascular y disminuyendo la morbilidad con sección muscular mínima.

El diseño utilizado en nuestro caso permite la reconstrucción del glande en tiempo quirúrgico, lo cual presenta ventajas como el menor costo y morbilidad comparado con otras técnicas como la de Norfolk, que requiere de dos tiempos quirúrgicos (1) o la realización de tatuajes para restaurar la apariencia deI glande (9). Otra ventaja importante es la ausencia de distorsión de la anatomía del área púbica (5), la similitud del color del área donante con la receptora (6) y el hecho de mantener la inervación de la isla cutánea, lo que teóricamente, es superior al colgajo antebraquial radial en el que por definición existen discrepancias en el tamaño de las fibras nerviosas a la hora de la anastomosis sensitiva (6). Sin embargo, algunos autores han reportado sensibilidad adecuada a pesar de requerirse anastomosis nerviosa y estar sujeto a la recuperación individual de cada paciente (9). En caso de una vasculatura y anatomía pélvica incierta, se prefiere el uso de un colgajo pediculado porque aporta su propio suministro de sangre al falo (9-10).

Tabla 1. Revisión de la literatura

\begin{tabular}{|c|c|c|c|}
\hline Autor & Número de casos & Longitud del colgajo & Sensibilidad \\
\hline $\begin{array}{l}\text { Pérez et al. } \\
(2005)\end{array}$ & 1 & $16 \times 18 \mathrm{~cm}$ & Sensibilidad táctil a los 6 meses de seguimiento \\
\hline $\begin{array}{l}\text { Mutaf et al. } \\
(2003)\end{array}$ & 1 & $14 \times 18 \mathrm{~cm}$ & $\begin{array}{l}\text {-Sensibilidad táctil (vibratoria) a los } 6 \text { meses de seguimiento } \\
\text {-Sensibilidad erógena a los } 12 \text { meses de seguimiento } \\
\text {-Se usó prótesis }\end{array}$ \\
\hline $\begin{array}{l}\text { Rubino et al. } \\
\text { (2007) }\end{array}$ & 1 & $16 \times 12 \mathrm{~cm}$ & $\begin{array}{l}\text { Sensibilidad táctil a los } 6 \text { meses de } \\
\text { seguimiento }\end{array}$ \\
\hline Lee et al. (2009) & 2 & $20 \times 16 \mathrm{~cm}$ & No reportado \\
\hline $\begin{array}{l}\text { Descamps et al. } \\
\text { (2009) }\end{array}$ & 3 & No especificado & No se realizó anastomosis nerviosa \\
\hline $\begin{array}{l}\text { Rashid et al. } \\
\text { (2011) }\end{array}$ & $\begin{array}{l}\text { Total de casos: } 14 \\
\text { Reconstrucción total: } 9 \\
\text { casos } \\
\text { Reconstrucción parcial: } \\
5 \text { casos }\end{array}$ & $\begin{array}{l}\text {-Faloplastia total: } \\
13 \times 14 \mathrm{~cm} \\
\text {-Faloplastia parcial: } \\
7 \times 8 \mathrm{~cm}\end{array}$ & $\begin{array}{l}\text { Todos los casos con sensibilidad táctil a los } 9 \text { meses de } \\
\text { seguimiento }\end{array}$ \\
\hline $\begin{array}{l}\text { Morrison et al. } \\
\text { (2014) }\end{array}$ & 1 & $12 \times 18 \mathrm{~cm}$ & Sensibilidad erógena a los 12 meses de seguimiento \\
\hline
\end{tabular}

A pesar de las ventajas descritas para el colgajo ALM, algunos autores señalan que el diseño del mismo debe ser meticuloso, pues se cuenta con poco espacio para alterar la orientación del colgajo (4). Aunque no utilizamos prótesis peneana en nuestro caso, consideramos que es importante la inclusión de la fascia lata como sustituto de la túnica albugínea, ya que permite, según múltiples autores, adicionar firmeza al colgajo (5), disminuir laxitud tisular (5) y fijar proximalmente la prótesis en caso de requerirlo, ya sea en el primer tiempo (3) o de forma diferida a los 12 meses, como prefieren realizarlo algunos autores por las altas tasas de retiro de las mismas (que puede alcanzar hasta un $50 \%)(10)$.

La principal dificultad en nuestro caso fue la presencia del tejido graso subcutáneo, que hizo difícil la creación del glande, aspecto reportado por varios autores (4) y para lo cual se ha propuesto adelogzar el tejido dejando $5 \mathrm{~mm}$ de grasa subcutánea (5). 
En este caso, el paciente no requirió reconstrucción formal de uretra ya que la longitud residual permitió su sutura al colgajo, ya que la reconstrucción uretral completa con el colgajo anterolateral, representa un escenario en el cual es más difícil la realización de la técnica tubo dentro de tubo; en este último caso es importante considerar el colgajo antebraquial radial como una opción válida, ya que el tejido es delgado y lo hace más maleable para este tipo de reconstrucción.

En conclusión, el colgajo ALM regional basado en perforantes, es una excelente opción para la realización de faloplastia parcial o total, ya que permite cumplir con los objetivos reconstructivos de forma segura, en un solo tiempo quirúrogico, sin requerir técnica microquirúrogica, con mínima morbilidad y una cicatriz ocultable que facilita la desestiomatización del procedimiento a futuro.

\section{CONFLICTOS DE INTERESES}

Ninguno por declarar.

\section{REFERENCIAS BIBLIOGRÁFICAS}

1. Morrison SD, Shakir A, Vyas KS, Kirby J, Crane CN, Lee GK. Phalloplasty: A Review of Techniques and Outcomes. Plast Reconstr Surg. 2016 Sep;138(3):594615. DOI 10.1097/PRS.0000000000002518.

2. Morrison SD, Son J, Song J, Berger A, Kirby J, Ahdoot $M$, et al. Modification of the tube-in-tube pedicled anterolateral thigh flap for total phalloplasty: the mushroom flap. Ann Plast Surg. 2014 May;72 Suppl 1:S226. DOI 10.1097/SAP.0000000000000072.
3. Mutaf M, Isik D, Bulut O, Büyükgüral B. A true onestage nonmicrosurgical technique for total phallic reconstruction. Ann Plast Surg. 2006 Jul;57(1):100-6.

4. Rashid M, Aslam A, Malik S, Tamimy MS, Ehtesham-ulHaq, Aman S, et al. Clinical applications of the pedicled anterolateral thigh flap in penile reconstruction. J Plast Reconstr Aesthet Surg. 2011 Aug;64(8):1075-81. DOI 10.1016/j.bjps.2011.01.009.

5. Casado Pérez C, Rodríguez Vegas JM, Ruiz Alonso E, Peña Alonso A. Faloplastia en un cambio de sexo de mujer a hombre con un colgajo sensible pediculado anterolateral de muslo. Cir Plast Iberolatinoam. 2005;31(3):175-81.

6. Rubino C, Figus A, Dessy LA, Alei G, Mazzocchi M, Trignano $\mathrm{E}$, et al. Innervated island pedicled anterolateral thigh flap for neo-phallic reconstruction in female-tomale transsexuals. J Plast Reconstr Aesthet Surg. 2009 Mar;62(3):e45-9. DOI 10.1016/j.bjps.2007.11.056.

7. Ascha M, Massie JP, Morrison SD, Crane CN, Chen ML. Outcomes of Single Stage Phalloplasty by Pedicled Anterolateral Thigh Flap versus Radial Forearm Free Flap in Gender Confirming Surgery. J Urol. 2018 Jan;199(1):206-14. DOI 10.1016/j.juro.2017.07.084.

8. Lee GK, Lim AF, Bird ET. A novel single-flap technique for total penile reconstruction: the pedicled anterolateral thigh flap. Plast Reconstr Surg. 2009 Jul;124(1):1636. DOI 10.1097/PRS.0b013e3181ab2593.

9. Descamps MJ, Hayes PM, Hudson DA. Phalloplasty in complete aphallia: pedicled anterolateral thigh flap. J Plast Reconstr Aesthet Surg. 2009 Mar;62(3):e51-4.

10. Lumen N, Monstrey S, Ceulemans P, van Laecke E, Hoebeke P. Reconstructive surgery for severe penile inadequacy: phalloplasty with a free radial forearm flap or a pedicled anterolateral thigh flap. Adv Urol. 2008:704343. DOI 10.1155/2008/704343. 